\title{
Agrosuppression: A Bioassay for the Hypersensitive Response Suited to High-Throughput Screening
}

\author{
Sophien Kamoun, Walid Hamada, and Edgar Huitema \\ Department of Plant Pathology, The Ohio State University, Ohio Agricultural Research and Development Center, \\ Wooster, $\mathrm{OH} 44691$, U.S.A.
}

Submitted 29 July 2002. Accepted 24 September 2002.

\begin{abstract}
We describe a novel method, agrosuppression, that addresses the need for an assay of the hypersensitive response (HR) in intact plants that is rapid and adapted to highthroughput functional screening of plant and pathogen genes. The agrosuppression assay is based on inoculation of intact plants with a mixture of Agrobacterium tumefaciens strains carrying (i) a binary plasmid with one or more candidate HR-inducing genes and (ii) a tumor-inducing (oncogenic) T-DNA. In the absence of HR induction, tumor formation is initiated, resulting in a typical crown gall phenotype. However, upon induction of the HR, tumor formation by the oncogenic T-DNA is suppressed, resulting in a phenotype that can be readily scored. We tested and optimized agrosuppression in Nicotiana benthamiana using the inf1 elicitin gene from the oomycete pathogen Phytophthora infestans, which specifically induces the $\mathrm{HR}$ in Nicotiana spp., and the gene-for-gene pair $A v r 9 / C f-9$ from the fungal pathogen Cladosporium fulvum and Lycopersicon pimpinellifolium (currant tomato), respectively. Agrosuppression protocols that can be rapidly performed using simple mechanical wounding of petioles of intact $N$. benthamiana plants were developed and appeared particularly adapted to intensive high-throughput screening. This assay promises to greatly facilitate the cloning of novel plant $\boldsymbol{R}$ genes and pathogen $A v r$ genes and to accelerate functional analyses and structure-function studies of these genes.
\end{abstract}

Additional keywords: agroinfiltration, disease resistance, transformation.

The hypersensitive response (HR) is a form of programmed cell death in plants that often accompanies defense responses to plant pathogens as diverse as viruses, bacteria, nematodes, fungi, and oomycetes (Beers and McDowell 2001; Dangl et al. 1996; Lam et al. 2001). Under natural conditions, the HR is tightly regulated, and specific signals are required for its activation. Such signals include a particular class of pathogen molecules, elicitors, that are perceived by plants resulting in the induction of the HR and resistance to the invading pathogen (Baker et al. 1997; Kjemtrup et al. 2000; Lauge and De Wit 1998). This model has been genetically defined by Flor's gene-for-gene hypothesis that a resistance reaction is determined by the simultaneous expression of a pathogen avirulence

Corresponding author: S. Kamoun; Department of Plant Pathology, 1680 Madison Ave.; Telephone: 330-263 3847; Fax: 330-263 3841; E-mail: kamoun.1@osu.edu or Avr gene, which typically encodes a specific elicitor, with the matching plant disease resistance or $R$ gene (Dangl and Jones 2001; Staskawicz et al. 1995). In recent years, the genefor-gene hypothesis has received tremendous experimental support through the identification and functional characterization of both $A v r$ and $R$ genes (Dangl and Jones 2001; Hammond-Kosack and Jones 1997; Kjemtrup et al. 2000; Lauge and De Wit 1998; Staskawicz et al. 1995).

Several experimental procedures have been used to assay whether a particular gene can induce the HR in plant cells and to help clone $A v r$ and $R$ genes. These include particle cobombardment with a construct expressing a reporter gene (Gopalan et al. 1996; Mindrinos et al. 1994), viral expression systems (Hammond-Kosack et al. 1995; Joosten et al. 1997; Kamoun et al. 1999a; Kooman-Gersmann et al. 1997; Lauge et al. 2000, 1998; Takken et al. 2000; Tampakaki and Panopoulos, 2000; Tobias et al. 1999), and Agrobacterium tumefaciens-based assays such as agroinfiltration or stable transformation (Bendahmane et al. 2000; Keller et al. 1999; Nimchuk et al. 2000; Tai et al. 1999; Van den Ackerveken et al. 1996; Van der Hoorn et al. 2000). However, despite their extensive use in cloning and functional analyses of $A v r$ and $R$ genes, these assays are not always adapted to the high-throughput functional screenings that are required for genomic research. For example, both particle bombardment and agroinfiltration require extensive sample preparation, and using these techniques to assay hundreds of clones simultaneously remains impractical. Some virus-based expression systems, particularly the binary potato virus $\mathrm{X}$ (PVX) system (Baulcombe 1999; Takken et al. 2000), are available in formats adapted to high-throughput screenings. However, PVX vectors typically cannot accommodate inserts larger than $2 \mathrm{~kb}$ or intron-containing gene sequences, thus preventing their use in assaying large fragments of cloned genomic DNA.

To overcome the limitations of the existing assays, we developed a novel method, agrosuppression, that addresses the need for an assay of the HR that is rapid and adapted to highthroughput functional screening of plant and pathogen genes. Agrosuppression is based on inoculations of intact plants with a mixture of $A$. tumefaciens strains carrying (i) a binary plasmid with one or more candidate test genes, and (ii) a tumor-inducing (oncogenic) T-DNA. In the absence of HR induction, tumor formation is initiated, resulting in a typical crown gall phenotype. However, upon induction of the HR, tumor formation by the oncogenic T-DNA is suppressed, resulting in a phenotype that can be readily scored. We validated and optimized agrosuppression in Nicotiana benthamiana using the infl elicitin gene from the oomycete pathogen Phytophthora infestans, 
which specifically induces the HR in Nicotiana spp. (Kamoun et al. 1997), and the gene-for-gene pair Avr9/Cf-9 from the fungal pathogen Cladosporium fulvum and Lycopersicon pimpinellifolium (currant tomato), respectively (Jones et al. 1994; Van den Ackerveken et al. 1992; van Kan et al. 1991). We anticipate that agrosuppression will significantly facilitate the cloning of novel plant $R$ genes and pathogen Avr genes and accelerate functional analyses and structure-function studies of these genes.

\section{RESULTS}

\section{Wound inoculations of $N$. benthamiana petioles with A. tumefaciens.}

Assays centered on wound inoculation of A. tumefaciens strains with toothpicks or similar mechanical wounding devices are well adapted to high-throughput screens. However, problems are encountered when clear-cut differences cannot be observed between various constructs, or symptoms cannot be reproduced reliably. For example, the phenotypes obtained following wound inoculations of petioles of $N$. benthamiana with A. tumefaciens carrying pInf1, a binary plasmid containing the $P$. infestans HR-inducing gene infl in an expression cassette, and with pGUSi, a control binary plasmid containing the $\beta$ glucuronidase gene interrupted by an intron, were not easy to distinguish (Fig. 1A and B). Petioles inoculated with A. tumefaciens carrying pINF1 displayed a necrotic ring and necrotic streaks along the veins. However, these symptoms were not consistently reproducible and were often difficult to discern from background damage associated with the bacterial inoculum and wounding. On the other hand, symptoms induced by A. tumefaciens carrying pINF1 or pGUSi were unambiguously different from the symptoms observed in petioles inoculated with the oncogenic supervirulent strain A281 (C58:pTiBo542) (Fig. 1C). Petioles inoculated with A281 showed, as early as five days after inoculation, typical cellular proliferation associated with tumor formation that often resulted in a marked deformation of the petiole (referred to as "crooked petiole phenotype").
Agrosuppression assay.

The distinct nature of the tumor phenotype led us to further investigate wound-inoculation assays involving oncogenic $A$. tumefaciens strains. We hypothesized that cell death induced by the transient expression of an $A v r$ or $R$ gene would impair the growth and development of oncogenic tissue to discernible levels, thus providing a distinct phenotypic difference. To test whether an A. tumefaciens carrying a binary vector expressing a HR-inducing gene can suppress the tumor phenotype induced by A281, we devised a series of inoculations with mixtures of $A$. tumefaciens strains. We inoculated petioles of $N$. benthamiana with the tumor-inducing strain A281 in 1:1 mixtures with A. tumefaciens carrying pINF1 or pGUSi (Fig. 2). Plants inoculated with the mixture of A281 and the pGUSi strain consistently showed tumor formation and the crooked petiole phenotype (Fig. 2A) similar to inoculations with A281 alone (Fig. 1C). In sharp contrast, plants inoculated with mixtures of A281 and the pInf1 strain did not form tumors or any notable deformation of the petioles (Fig. 2B). To further confirm that no tumor growth was occurring in mixed inoculations between A281 and the pInf1 strain, we examined representative inoculated petioles with scanning electron microscopy (Fig. 3). No evidence of tumor formation was observed, in contrast to the extensive cell proliferation and tissue deformation observed in petioles inoculated with mixtures of A281 and the pGUSi strain. These results suggest that the cell death response induced by the pInf1 construct suppressed tumor formation by wild-type A. tumefaciens in trans, resulting in a distinct phenotype. Consequently, we named this assay suppression of Agrobacterium tumor formation, or agrosuppression.

To test the efficiency of agrosuppression in large-scale experiments, we assayed $289 \mathrm{~N}$. benthamiana petioles with 1:1 mixtures of pInf1 and pGUSi strains, pInf1 and A281 strains, as well as A281 and pGUSi strains. All observed symptoms were consistent with the results shown in Figures 1 and 2, except for one petiole inoculated with the mixture of pInf1 and A281 strains, which showed tumor formation instead of the predicted tumor suppression. These results suggest that
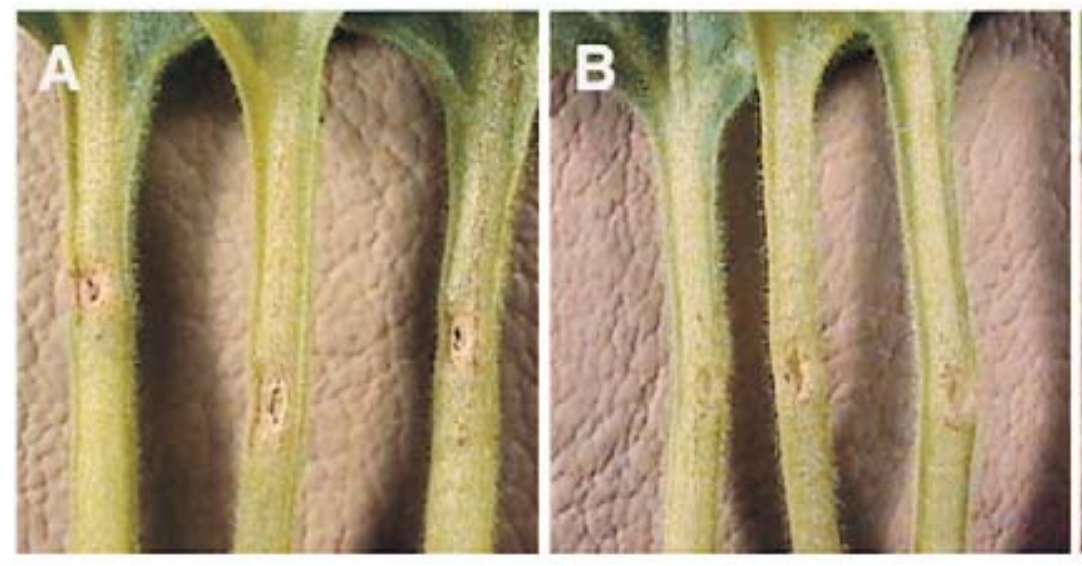

D

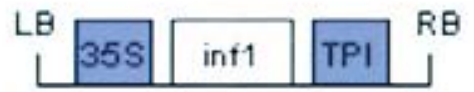

E

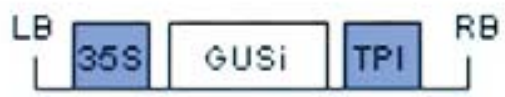

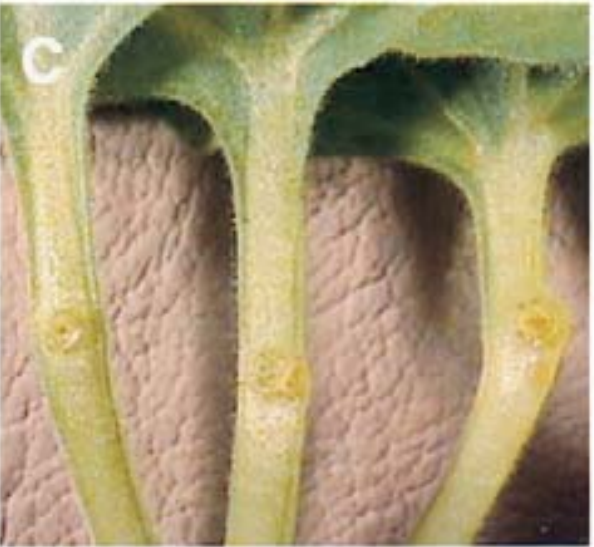

$\mathbf{F}$

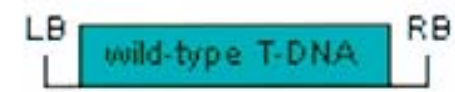

Fig. 1. Symptoms observed in petioles of Nicotiana benthamiana following inoculations with Agrobacterium tumefaciens strains. N. benthamiana petioles 14 days after inoculation with $\mathbf{A}$, A. tumefaciens strains carrying pInf1 (induces the hypersensitive response in Nicotiana spp.) or B, pGUSi (negative control) and C, supervirulent A. tumefaciens strain A281 (C58:pTiBo542, induces crown gall tumors). For clarity, the relevant elements of the T-DNAs are schematically represented for D, pInf1, E, pGUSi, and F, pTiBo542. 35S refers to the Cauliflower mosaic virus (CaMV) $35 \mathrm{~S}$ promoter and PI refers to the potato proteinase-II terminator region. Three representative petioles are shown for each treatment. 
the rates of false positives and false negatives for agrosuppression are below $1 \%$ and are acceptable for large-scale screening.

\section{Reconstruction of the Avr9/Cf-9 gene} for gene interaction using agrosuppression.

To test whether we could reconstruct a gene-for-gene quadratic check using agrosuppression, we inoculated $N$. benthamiana with $A$. tumefaciens strains carrying pAvr9, a binary plasmid containing the C. fulvum avirulence gene Avr9 in an expression cassette, pCf9, a binary plasmid containing the tomato $R$ gene $C f-9$ in an expression cassette, or pGUSi mixed with the oncogenic A281 in four different combinations (Fig. 4). Plants inoculated with the combination of A281 (50\%), pAvr9 strain $(25 \%)$, and pCf9 strain $(25 \%)$ failed to show tumors or deformation of the petioles. On the other hand, plants inoculated with the three other combinations, in which at least one of the matching genes was missing, consistently showed tumors or crooked petioles, or both. These results are consistent with the gene-for-gene model and indicate that agrosuppression occurred only when the matching $A v r 9$ and $C f-9$ genes were simultaneously expressed and the HR was expected to occur.

\section{Comparison of the agrosuppression and agroinfiltration assays for the elicitin-like genes inf4 and inf6.}

To compare the agrosuppression assay with the well-established agroinfiltration assay, we tested two elicitin-like genes, inf4 and inf6, which were recently described from $P$. infestans and have not been previously tested for induction of the HR (Kamoun et al. 1999b). We first applied the agroinfiltration protocol and injected leaves of $N$. benthamiana with A. tumefaciens strains carrying pInf4, a binary plasmid containing the $P$. infestans gene inf4 in an expression cassette, or pInf6, a binary plasmid containing the $P$. infestans gene inf6 in an expression cassette. Leaves injected with the pInf6 strain induced a confluent necrosis typical of the HR, whereas leaves injected with the pInf4 strain failed to show visible symptoms (data not shown). We then inoculated petioles of $N$. benthamiana with the tumor inducing strain A281 in 1:1 mixtures with A. tumefaciens carrying pINF4 or pINF6. In contrast to plants inoculated with the mixture of A281 with the pInf6 strain, plants inoculated with the mixture of A281 with the pInf4 strain consistently showed the tumor phenotype. These results confirm that the agrosuppression assay is reliable and yields similar results to the widely used agroinfiltration assay (Table 1).

\section{Quantification of tumor suppression}

by a dilution series of $A$. tumefaciens carrying pInf1.

To determine whether diluted inoculations of A. tumefaciens carrying pInf1 can still result in suppression of tumor formation by oncogenic $A$. tumefaciens, we inoculated $N$. benthamiana plants with various ratios of the pInf1 strain mixed to A281. The percentage of inoculated petioles showing suppression of tumor formation was counted 10 to 16 days postinoculation and was plotted against the ratio of the pInf1 strain (Fig. 5). The percentage of pInf1 strain that resulted in $50 \%$ suppression

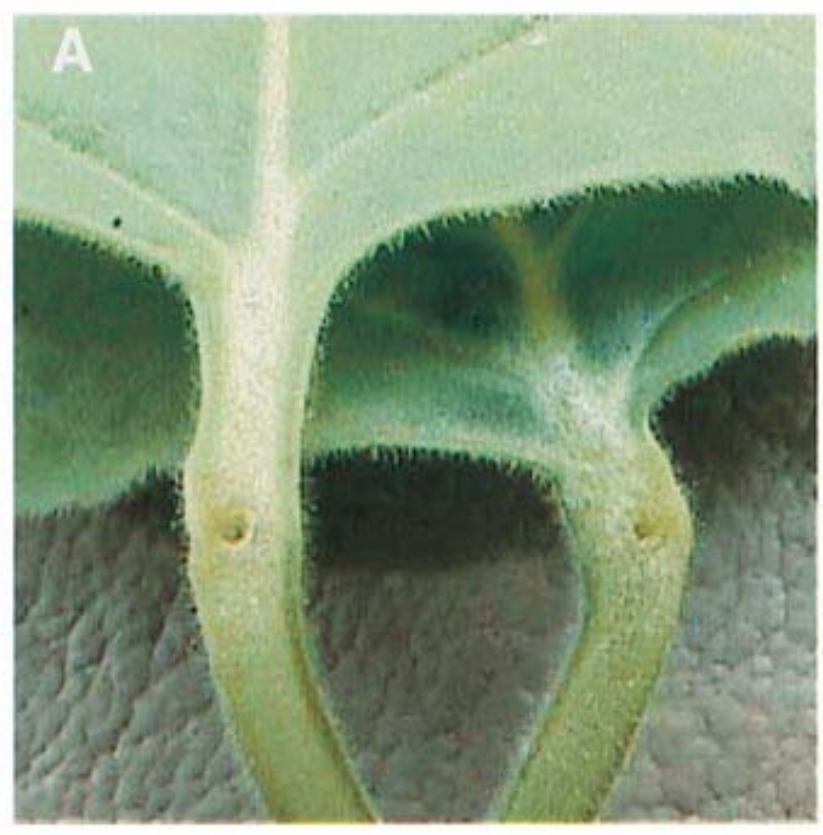

C

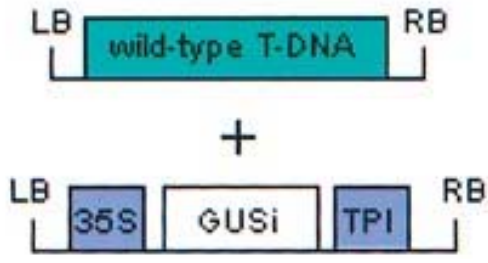

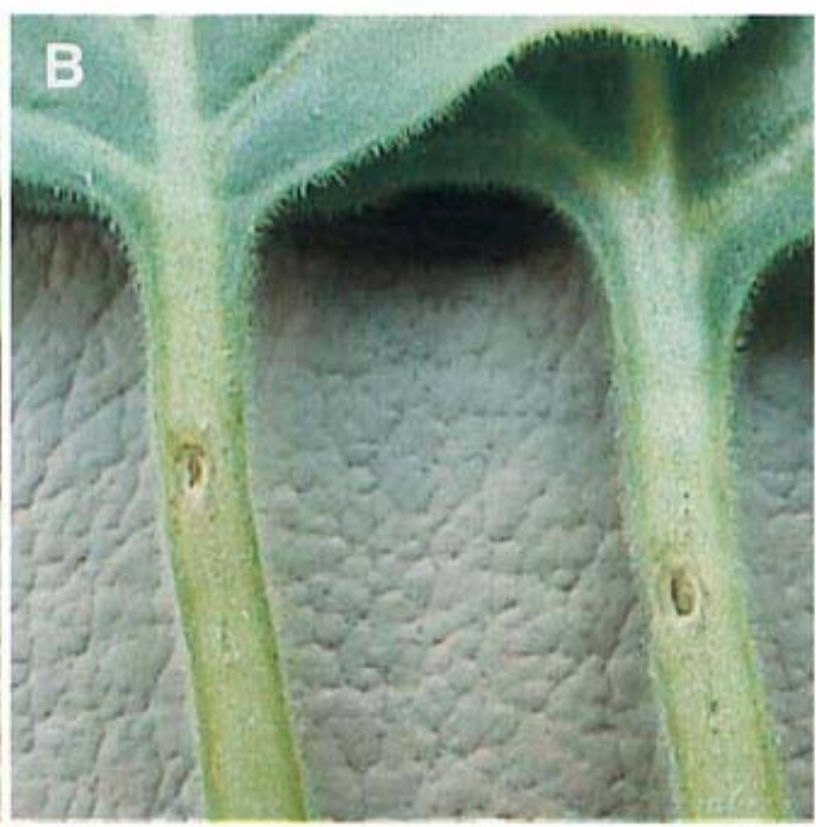

D

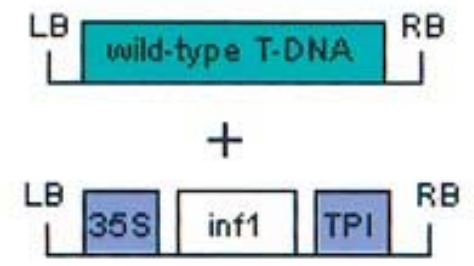

Fig. 2. Symptoms observed in petioles of Nicotiana benthamiana following inoculations with mixtures of Agrobacterium tumefaciens strains. A, N. benthamiana petioles 14 days after inoculation with A. tumefaciens oncogenic strain A281 (C58:pTiBo542) mixed with an A. tumefaciens strain carrying pGUSi, or B, mixed with an A. tumefaciens strain carrying pInf1. C, For clarity, the relevant elements of the T-DNAs are schematically represented for pTiBo542 and pGUSi, and D, pTiBo542 and pInf1. All mixtures were made in 1:1 ratios. 35S refers to the Cauliflower mosaic virus (CaMV) $35 \mathrm{~S}$ promoter and PI refers to the potato proteinase-II terminator region. Two representative petioles are shown for each treatment. 
of tumor formation was calculated to be $0.6 \%$ and $0.8 \%$ from two independent experiments. At dilutions of $20 \%$ or higher of the pInf1 strain, $100 \%$ suppression of tumor formation was observed at all inoculation sites. These results indicate that suppression of tumor formation can still be observed at relatively low dilutions of the A. tumefaciens strain carrying pInf1 and suggest that assays that involve pooling multiple $A$. tumefaciens strains could be considered.

\section{DISCUSSION}

In this paper, we describe a novel method, agrosuppression, that addresses the need for an assay of the HR that is rapid and adapted to high-throughput functional screening of cloned plant and pathogen genes. This assay is based on the observation that cell death is dominant in trans over oncogenicity. Therefore, coinoculation of plants with a mixture of two A. tumefaciens strains, one carrying a binary plasmid with a HR-inducing gene and the other carrying an oncogenic Ti plasmid, results in suppression of the crown galls induced by the oncogenic strain. Agrosuppression fulfills the requirements for high-throughput screening, including rapid preparation and inoculation of samples, testing of a large number of samples per experiment, and easy visual scoring of symptoms. We validated and optimized the agrosuppression assay using cloned $A v r$ genes from the oomycete pathogen $P$. infestans and the fungal pathogen $C$. fulvum, as well as the $R$ gene $C f-9$ from tomato.

In principle, the agrosuppression assay can be applied to a variety of plant tissues. However, we found that wound inoculations of $N$. benthamiana petioles using toothpicks is the most rapid and reliable inoculation procedure that is also suited to large-scale screenings. Approximately five inoculations can be performed per plant, suggesting that hundreds of assays can be performed with only minimal square footage of greenhouse space. The suppression of tumor formation can be easily distin-
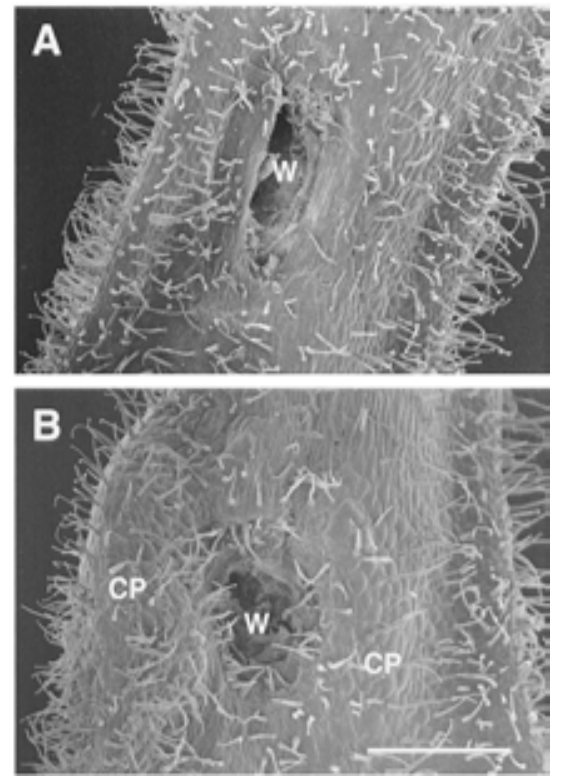

Fig. 3. Scanning electron microscopy of petioles of Nicotiana benthamiana following inoculations with mixtures of Agrobacterium tumefaciens strains. $N$. benthamiana petioles 14 days after inoculation with $A$. tumefaciens oncogenic strain A281 (C58:pTiBo542) mixed with A, an A. tumefaciens strain carrying pInf1 or $\mathbf{B}$, mixed with an A. tumefaciens strain carrying pGUSi were dehydrated and examined using high vacuum scanning electron microscopy. W refers to the wound inoculation point and $\mathrm{CP}$ refers to the areas of cellular proliferation typical of crown galls. The bar corresponds to $1 \mathrm{~mm}$. guished from emerging tumors within 7 to 15 days after inoculation, using visual inspection or image analysis software. Scoring plants is facilitated by the inoculation of petioles of $N$. benthamiana, in which emerging tumors cause a deformation that results in the crooked petiole phenotype (Figs. 1 and 2).

We optimized the agrosuppression assay in N. benthamiana, a solanaceous plant well known as a favorable host for both $A$. tumefaciens transformation and virus infection. However, in principle, the assay could be expanded to other plant species since the supervirulent $A$. tumefaciens strain A281 (chromosomal background from $\mathrm{C} 58$ with $\mathrm{Ti}$ plasmid pTiBo542) is known to induce tumors on a wide range of host plants (Jin et al. 1987). Indeed, preliminary results from our laboratory indicate that agrosuppression can also be successfully applied to tomato (data not shown).

The agrosuppression phenomenon suggests that a high proportion of transformed cells in plants inoculated with combinations of A. tumefaciens strains contain the mixture of T-DNAs. Rezmer and associates (1999) recently used GUS assays and reverse transcriptase polymerase chain reaction (RT-PCR) to demonstrate that most, if not all, cells in tumors induced by $A$. tumefaciens contain a copy of the T-DNA. These results are concordant with the observation that crown gall tissue contains organized networks of vascular bundles with phloem and xylem functionally connected to the vascular system of the host and, therefore, is unlikely to be formed of an uncoordinated maze of mosaic cells (Rezmer et al. 1999). Consistent with these observations, histochemical GUS assays of crown galls obtained from $N$. benthamiana plants inoculated with a mixture of the pGUSi strain and oncogenic strain A281 (Fig. 2A) revealed that a significant proportion of the tumor tissue expresses the gus gene (data not shown). In any case, one can easily avoid using mixtures of $A$. tumefaciens strains in the agrosuppression assay by introducing a compatible set of binary and oncogenic Ti plasmids into a single $A$. tumefaciens strain. Such an approach should further simplify the agrosuppression procedure.

We compared agrosuppression with agroinfiltration, an assay that became widely used in recent years for transient expression of $A v r$ and $R$ genes in plant cells (Bendahmane et al. 2000; Kapila et al. 1997; Nimchuk et al. 2000; Tai et al. 1999; Van den Ackerveken et al. 1996; Van der Hoorn et al. 2000). The two methods yielded consistent results. A. tumefaciens con-

Table 1. Symptoms observed in Nicotiana benthamiana with the agroinfiltration and agrosuppression assays using the various constructs tested in this study ${ }^{\mathrm{a}}$

\begin{tabular}{|c|c|c|}
\hline $\begin{array}{l}\text { A. tumefaciens binary } \\
\text { plasmid }\end{array}$ & Agroinfiltration & Agrosuppression \\
\hline \multicolumn{3}{|l|}{ P. infestans constructs } \\
\hline pInf1 & HR & NVS \\
\hline pInf4 & NVS & Tumors \\
\hline pInf6 & HR & NVS \\
\hline \multicolumn{3}{|c|}{ C. fulvum-L.pimpinellifollium constructs } \\
\hline pAvr9 + pCf 9 & HR & NVS \\
\hline pAvr9 + pGUSi & NVS & Tumors \\
\hline pCf9 + pGUSi & NVS & Tumors \\
\hline \multicolumn{3}{|l|}{ Control construct } \\
\hline pGUSi & NVS & Tumors \\
\hline
\end{tabular}

${ }^{a}$ Symptoms were observed following injection of fully expanded $N$. benthamiana leaves with Agrobacterium tumefaciens strains carrying the various binary plasmid construct (agroinfiltration) or following wound inoculation of $N$. benthamiana petioles with the A. tumefaciens binary plasmid strain mixed with the A. tumefaciens oncogenic strain A281 (agrosuppression). HR refers to the hypersensitive response, NVS to no visible symptoms, and tumors to crown galls or petiole deformation, or both. Results of the agroinfiltration assays with pAvr9 and pCf9 were obtained from van der Hoorn and associates (2000). 
structs that resulted in induction of the HR following injection into leaf panels were always able to induce suppression of tumor formation in petioles (Table 1). These results included assays with inf4 and inf6, two novel $P$. infestans elicitin-like genes that were not previously tested for HR induction (Kamoun et al. 1999b). Since the same A. tumefaciens binary plasmid constructs and strains can be used for both agroinfiltration and agrosuppression, the two assays can be combined or used interchangeably depending on the needs of a particular experiment. Agroinfiltration is more suitable in experiments involving a small number of constructs and might be considered more reliable since the HR phenotype is assessed directly. However, agrosuppression is better suited to experiments that require handling of a large number of constructs, such as in highthroughput screening of candidate and random genes. Notably, in contrast to agroinfiltration, agrosuppression does not require acetosyringone induction of Agrobacterium cultures or syringe infiltration of leaf panels, two laborious steps that limit the use of agroinfiltration in large-scale experiments. Nonetheless, the two assays can be used sequentially in a two-step strategy. A given clone library is screened first in a high-throughput setting using agrosuppression, then all positives emerging out of the primary screen are retested using agroinfiltration.

The agrosuppression method has exciting potential for facilitating the cloning of plant $R$ genes and pathogen $A v r$ genes and the functional characterization of gene-for-gene interactions. One particularly attractive application is in functional screening of plant genomic libraries and candidate genomic clones for the presence of $R$ genes. Indeed, clones from genomic libraries constructed in binary plasmid vectors, including binary bacterial artificial chromosomes (BI-BACs) (Hamilton 1997; Hamilton et al. 1996) and transformation-competent bacterial artificial chromosomes (TAC vectors) (Liu et al. 1999, 2000; Shibata and Liu 2000) can be directly introduced into appropriate $A$. tumefaciens strains and assayed for the induction of the HR by coinoculation with an A. tumefaciens strain carrying a binary plasmid with the matching $A v r$ gene. Since agrosuppression requires little sample preparation, hundreds of independent clones can be assayed concurrently, and significant portions of a plant genome can be tested. In addition, diluted inoculations with the pInf1 strain suggest that a pooling strategy in which multiple $A v r$ constructs are screened simultaneously might be feasible. Another application of agrosuppression is in structure-function analyses of cloned $R$ genes, in particular agrosuppression should greatly facilitate screening for gain-offunction mutants of $R$ genes that constitutively induce the HR in the absence of the matching Avr gene (Rathjen et al. 1999). In summary, we anticipate that agrosuppression will find many applications in the study of gene-for-gene interactions and will be an effective tool for functional genomics of plant-pathogen interactions.

\section{MATERIALS AND METHODS}

\section{DNA manipulations.}

DNA manipulations were conducted essentially as described elsewhere (Sambrook et al. 1989).

\section{Plasmid constructions.}

Plasmids pInf1, pInf4, and pInf6 were constructed by cloning PCR amplified DNA fragments corresponding to a fusion between the signal sequence of the $P R-1 a$ gene of tobacco (Hammond-Kosack et al. 1995) and the sequence of the 98 amino-acid elicitin domain of INF1, INF4, and INF6 (Kamoun et al. 1997, 1999b), into pAvr9 (Van der Hoorn et al. 2000) digested with $N c o I$ and $S a c$ I to remove the Avr 9 open reading frame (ORF). The construction of the PR-1a::infl fusion was described elsewhere (Kamoun et al. 1999a). We used a similar overlap extension strategy to generate the PRla::inf4 and PRla::inf6 fusions. The overlapping oligonucleotides used in the PCRs were PR-INF4F (5'-CTTGCCGTGCCGCGTGCACGGCAAAACAAC-3') and PR-INF4R (5'-GCCGTGCACGCGGCGGCACGGCAAGAGTGGGATATTAC-3') for inf4, and PR-INF6F (5'-CTTGCCGTGCCGCCGCCTGCACGACTGCCCAG-3') and PR-INF6R (5'-GGCAGTCGTGCAGGCGGCGGCACGGCAAGAGTGGGATATTAC-3') for inf6. The three $P R-1 a:$ inf constructs were PCR-amplified with PR1-FNCO (5'-GCATCCATGGGATTTGTTCTCTTTTCACAA-3') as a forward primer and INF1-RSAC (5'-GGCGAGCTCTCATAGCGACGCACACGTAG-3'), INF4-RSAC (5'-GTGGAGCTCTTAAAGCCTCTTACAGTCAGAAGAGAAA-3'), and INF6RSAC (5'-GGGAAGAGCTCTCACAAGGCATCGCACTGCGTCT-3') as reverse primers for infl, inf4, and inf6, respectively, digested with $\mathrm{NcoI}$ and $\mathrm{SacI}$ restriction enzymes (restriction sites underlined) and ligated to pAvr9. The resulting pInf plasmids were confirmed by DNA sequencing to contain intact PR1a::inf ORFs flanked by the Cauliflower mosaic virus (CaMV) 35S promoter and the omega Tobacco mosaic virus (TMV) leader on the $5^{\prime}$ side, and the potato proteinase-II terminator region on the $3^{\prime}$ end. Plasmids pAvr9, pCf9, and pGUSi were described elsewhere (Van der Hoorn et al. 2000).

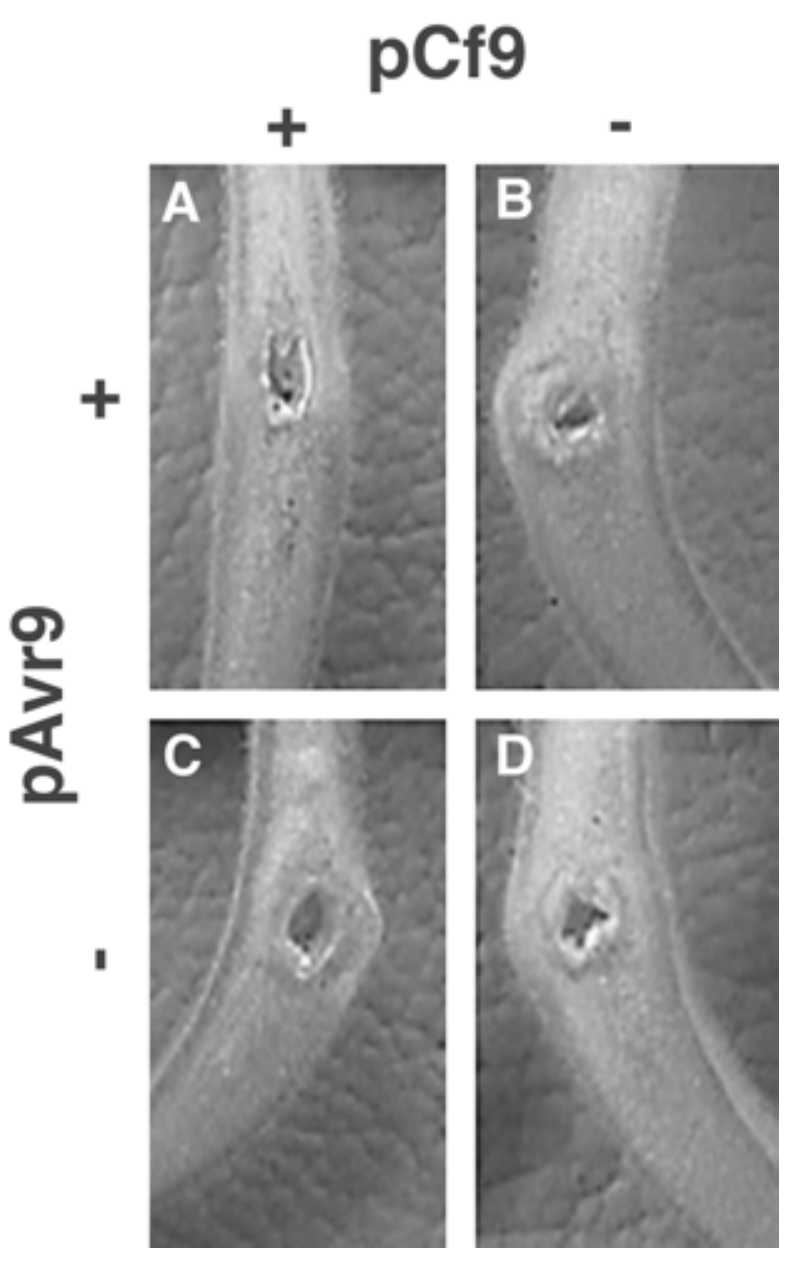

Fig. 4. Reconstruction of the Avr $9 / C f-9$ gene-for-gene interaction using agrosuppression. $N$. benthamiana petioles 16 days after inoculation with A. tumefaciens oncogenic strain A281 (C58:pTiBo542) mixed with A, A. tumefaciens strain carrying pAvr9 (25\% of total mixture) and another $A$. tumefaciens strain carrying pCf9 (25\%), B, pAvr9 strain (25\%) and an A. tumefaciens strain carrying pGUSi $(25 \%), \mathbf{C}$, pCf9 strain $(25 \%)$ and pGUSi strain (25\%), and D, pGUSi strain (50\%). 


\section{A. tumefaciens strains and culture conditions.}

pINF1, pINF4, and pINF6 were introduced by electroporation into A. tumefaciens strain MOG101 (Hood et al. 1993), whereas pAvr9 and pCf9 were introduced into A. tumefaciens strain GV3101 (Holsters et al. 1980). The supervirulent strain A281 (chromosomal background from C58 with Ti plasmid pTiBo542), which induces large, rapidly appearing tumors and has a wider host range than other $A$. tumefaciens strains (Jin et al. 1987), was used in the agrosuppression experiments. A. tumefaciens strains were routinely grown at $28^{\circ} \mathrm{C}$ in Luria-Bertani (LB) media using the appropriate antibiotics (Sambrook et al. 1989). Bacterial inoculum was prepared by suspending cultures grown for 2 days on solid LB agar plates in liquid LB and adjusting the concentration to an approximate optical density at $600 \mathrm{~nm}$ of 0.3 to 0.4 . Whenever necessary, different strains were combined in the desired ratios by mixing the adjusted suspensions.

\section{Agrosuppression assays.}

Young $N$. benthamiana plants at approximately the 10 - to 12-leaf stage (approximately 4-week-old) were used for the agrosuppression assays. Plants were cultured and maintained in a greenhouse with an ambient temperature of 22 to $25^{\circ} \mathrm{C}$ and high light intensity. Under these conditions, plants grew vigorously, which resulted in rapid formation of tumors. Inoculations were performed on the upper (younger) five leaves by dipping a wooden sterile toothpick in a bacterial suspension and wounding the plant once in the middle of the petiole. Inoculation of older (senescing or yellowing) leaves did not result in consistent tumor formation and was avoided. Symptoms were scored daily and typically started developing within a week after inoculation. All experiments were repeated at least two times, and a minimum of four plants or 20 petioles were inoculated per experiment and per treatment.

\section{Agroinfiltration assays.}

Recombinant $A$. tumefaciens strains containing the various binary plasmids were prepared for agroinfiltration as previ-

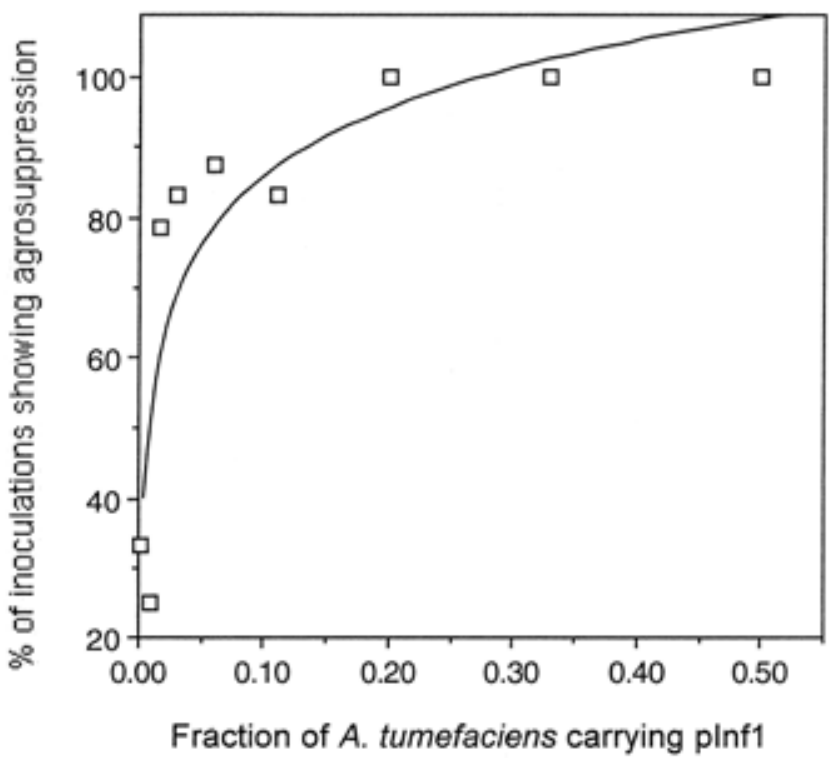

Fig. 5. Quantification of tumor suppression by a dilution series of Agrobacterium tumefaciens carrying pInf1. Various ratios of the pInf1 strain mixed to the oncogenic strain A281 (0.24 to 50\%) were wound inoculated on $N$. benthamiana plants and examined for suppression of tumor formation. Each time point corresponds to at least five independent inoculations. The graph illustrates one representative experiment out of two that were performed. ously described (Kapila et al. 1997; Van der Hoorn et al. 2000). Cultures were infiltrated into young and fully expanded leaves of $N$. benthamiana. Infiltrations were conducted side by side and were repeated at least three times.

\section{Scanning electron microscopy.}

High vacuum scanning electron microscopy of dehydrated petioles was performed using a Hitachi $3500 \mathrm{~N}$ scanning electron microscope. Samples were first fixed in $3 \%$ glutaraldehyde, $2 \%$ paraformaldehyde, and $0.1 \mathrm{M}$ potassium phosphate buffer, $\mathrm{pH} 7.4$, for at least $24 \mathrm{~h}$, and subsequently rinsed in distilled water for $15 \mathrm{~min}$. Samples were dehydrated through washes in 50, 75, 95, and $100 \%$ ethanol for 15 min each. Following the dehydration procedure, samples were dried in a critical-point dryer before mounting and viewing.

\section{ACKNOWLEDGMENTS}

We thank J. Finer for wild-type A. tumefaciens strains, R. Van der Hoorn and P. de Wit for pAvr9 and pCf9, T. Meulia and the staff of the OARDC Molecular and Cellular Imaging Center for help with electron microscopy, as well as S. Dong and D. Kinney for technical assistance. This work was supported by the OARDC Research Enhancement Grant Program and by Syngenta, Inc. Salaries and research support were provided by state and federal funds appropriated to the Ohio Agricultural Research and Development Center, the Ohio State University.

\section{LITERATURE CITED}

Baker, B., Zambryski, P., Staskawicz, B., and Dinesh-Kumar, S. P. 1997. Signaling in plant-microbe interactions. Science 276:726-733.

Baulcombe, D. C. 1999. Fast forward genetics based on virus-induced gene silencing. Curr. Opin. Plant Biol. 2:109-113.

Beers, E. P., and McDowell, J. M. 2001. Regulation and execution of programmed cell death in response to pathogens, stress and developmental cues. Curr. Opin. Plant Biol. 4:561-567.

Bendahmane, A., Querci, M., Kanyuka, K., and Baulcombe, D. C. 2000. Agrobacterium transient expression system as a tool for the isolation of disease resistance genes: Application to the $R x 2$ locus in potato. Plant J. 21:73-81.

Dangl, J. L., and Jones, J. D. 2001. Plant pathogens and integrated defence responses to infection. Nature 411:826-833.

Dangl, J. L., Dietrich, R. A., and Richberg, M. H. 1996. Death don't have no mercy: Cell death programs in plant-microbe interactions. Plant Cell 8:1793-1807.

Gopalan, S., Bauer, D. W., Alfano, J. R., Loniello, A. O., He, S. Y., and Collmer, A. 1996. Expression of the Pseudomonas syringae avirulence protein AvrB in plant cells alleviates its dependence on the hypersensitive response and pathogenicity (Hrp) secretion system in eliciting genotype-specific hypersensitive cell death. Plant Cell 8:1095-1105.

Hamilton, C. M. 1997. A binary-BAC system for plant transformation with high-molecular-weight DNA. Gene 200:107-116.

Hamilton, C. M., Frary, A., Lewis, C., and Tanksley, S. D. 1996. Stable transfer of intact high molecular weight DNA into plant chromosomes. Proc. Natl. Acad. Sci. U.S.A. 93:9975-9979.

Hammond-Kosack K. E., and Jones J. D. G. 1997. Plant disease resistance genes. Annu. Rev. Plant Physiol. Plant Mol. Biol. 48:575-607.

Hammond-Kosack K. E., Staskawicz B. J., Jones J. D. G., and Baulcombe D. C. 1995. Functional expression of a fungal avirulence gene from a modified potato virus X genome. Mol. Plant-Microbe Interact. 8:181-185.

Holsters, M., Silva, B., Van Vliet, F., Genetello, C., De Block, M. Dhaese, P., Depicker, A., Inze, D., Engler, G., Villaroel, R., Van Montagu, M., and Schell, J. 1980. The functional organization of the nopaline A. tumefaciens plasmid pTiC58. Plasmid 3:212-230.

Hood E. E., Gelvin S. B., Melchers L. S. and Hoekema A. 1993. New Agrobacterium helper plasmids for gene transfer to plants. Transgenic Res. 2:208-218.

Jin S. G., Komari T., Gordon M. P., and Nester E. W. 1987. Genes responsible for the supervirulence phenotype of Agrobacterium tumefaciens A281. J. Bacteriol. 169:4417-4425.

Jones D. A., Thomas C. M., Hammond-Kosack K. E., Balint-Kurti P. J., and Jones J. D. 1994. Isolation of the tomato $C f-9$ gene for resistance to Cladosporium fulvum by transposon tagging. Science 266:789-793.

Joosten M. H. A. J., Vogelsang R., Cozijnsen T. J., Verberne M. C., and 
de Wit P. J. G. M. 1997. The biotrophic fungus Cladosporium fulvum circumvents $\mathrm{Cf}-4$ mediated resistance by producing unstable AVR4 elicitors. Plant Cell 9:367-379.

Kamoun S., van West P., de Jong A. J., de Groot K., Vleeshouwers V. G. A. A., and Govers F. 1997. A gene encoding a protein elicitor of Phytophthora infestans is down-regulated during infection of potato. Mol. Plant-Microbe Interact. 10:13-20.

Kamoun S., Honée G., Weide, R., Laugé, R., Kooman-Gersmann M., de Groot K., Govers F. and de Wit P. J. G. M. 1999a. The fungal gene Avr 9 and the oomycete gene infl confer avirulence to potato virus $\mathrm{X}$ on tobacco. Mol. Plant-Microbe Interact. 12:459-462.

Kamoun S., Hraber, P., Sobral, B., Nuss, D. and Govers F. 1999b. Initial assessment of gene diversity for the oomycete pathogen Phytophthora infestans based on expressed sequences. Fun. Genet. Biol. 28:94-106.

Kapila J., de Rycke, R., Van Montagu, M., and Angenon, G. 1997. An Agrobacterium-mediated transient gene expression system for intact leaves. Plant Sci. 122:101-108.

Keller H., Pamboukdjian, N., Ponchet, M., Poupet, A., Delon, R., Verrier, J. L., Roby, D., and Ricci, P. 1999. Pathogen-induced elicitin production in transgenic tobacco generates a hypersensitive response and nonspecific disease resistance. Plant Cell 11:223-236.

Kjemtrup S., Nimchuk Z., and Dangl J. L. 2000. Effector proteins of phytopathogenic bacteria: Bifunctional signals in virulence and host recognition. Curr. Opin. Microbiol. 3:73-78.

Kooman-Gersmann M., Vogelsang, R., Hoogendijk, E. C. M., and de Wit, P. J. G. M. 1997. Assignment of amino acid residues of the Avr9 peptide of Cladosporium fulvum that determine elicitor activity. Mol. Plant-Microbe Interact. 10:821-829.

Lam E., Kato N., and Lawton M. 2001. Programmed cell death, mitochondria and the plant hypersensitive response. Nature 411:848-853.

Lauge R., and De Wit P. J. G. M. 1998. Fungal avirulence genes: Structure and possible functions. Fungal Genet. Biol. 24:285-297.

Lauge, R., Joosten, M. H. A. J., Haanstra, J. P., Goodwin, P. H., Lindhout, P., and De Wit, P. J. G. M. 1998. Successful search for a resistance gene in tomato targeted against a virulence factor of a fungal pathogen. Proc Natl. Acad. Sci. U.S.A. 95:9014-9018.

Lauge R., Goodwin P. H., De Wit P. J. G. M., and Joosten M. H. A. J. 2000. Specific HR-associated recognition of secreted proteins from Cladosporium fulvum occurs in both host and non-host plants. Plant J., 23:735-745.

Liu Y. G., Shirano, Y., Fukaki, H., Yanai, Y., Tasaka, M., Tabata, S., and Shibata, D. 1999. Complementation of plant mutants with large genomic DNA fragments by a transformation-competent artificial chromosome vector accelerates positional cloning. Proc. Natl. Acad. Sci. U.S.A. 96:6535-6540.

Liu Y. G., Nagaki, K., Fujita, M., Kawaura, K., Uozumi, M., and Ogihara, Y. 2000. Development of an efficient maintenance and screening system for large-insert genomic DNA libraries of hexaploid wheat in a transformation-competent artificial chromosome (TAC) vector. Plant $\mathbf{J}$. 23:687-695

Mindrinos, M., Katagiri, F., Yu, G. L., and Ausubel, F. M. 1994. The A. thaliana disease resistance gene RPS2 encodes a protein containing a nucleotide-binding site and leucine-rich repeats. Cell 78:1089-1099.

Nimchuk, Z., Marois, E., Kjemtrup, S., Leister, R. T., Katagiri, F., and Dangl, J. L. 2000. Eukaryotic fatty acylation drives plasma membrane targeting and enhances function of several type III effector proteins from Pseudomonas syringae. Cell 101:353-363.

Rathjen, J. P., Chang, J. H., Staskawicz, B. J., and Michelmore, R. W. 1999. Constitutively active Pto induces a Prf-dependent hypersensitive response in the absence of avrPto. Embo (Eur. Mol. Biol. Organ.) J 18:3232-3240.

Rezmer, C., Schlichting, R., Wachter, R., and Ullrich, C. I. 1999. Identification and localization of transformed cells in Agrobacterium tumefaciens-induced plant tumors. Planta 209:399-405.

Sambrook, J., Fritsch, E. F., and Maniatis, T. 1989. Molecular Cloning: A Laboratory Manual. (2nd ed.). Cold Spring Harbor Laboratory Press, Cold Spring Harbor, NY, U.S.A.

Shibata, D., and Liu, Y. G. 2000. Agrobacterium-mediated plant transformation with large DNA fragments. Trends Plant. Sci. 5:354-357.

Staskawicz, B. J., Ausubel, F. M., Baker, B. J., Ellis, J. G., and Jones, J. D. G. 1995. Molecular genetics of plant disease resistance. Science 268:661-667.

Tai, T. H., Dahlbeck, D., Clark, E. T., Gajiwala, P., Pasion, R., Whalen, M. C., Stall, R. E., and Staskawicz, B. J. 1999. Expression of the Bs2 pepper gene confers resistance to bacterial spot disease in tomato. Proc. Natl. Acad. Sci. U.S.A. 96:14153-14158.

Takken, F. L., Luderer, R., Gabriels, S. H., Westerink, N., Lu, R., De Wit, P. J. G. M. and Joosten, M. H. A. J. 2000. Technical advance: A functional cloning strategy, based on a binary PVX- expression vector, to isolate HR-inducing cDNAs of plant pathogens. Plant J. 24:275-283.

Tampakaki, A. K., and Panopoulos, N. J. 2000. Elicitation of hypersensitive cell death by extracellularly targeted $\mathrm{HrpZ}_{\mathrm{Psph}}$ produced in planta. Mol. Plant-Microbe Interact. 13:1366-1374.

Tobias, C. M., Oldroyd, G. E., Chang, J. H., and Staskawicz, B. J. 1999. Plants expressing the Pto disease resistance gene confer resistance to recombinant PVX containing the avirulence gene AvrPto. Plant J. 17:41-50.

Van den Ackerveken, G. F. J. M., van Kan, J. A. L., and de Wit, P. J. G. M. 1992. Molecular analysis of the avirulence gene avr9 of the fungal tomato pathogen Cladosporium fulvum fully supports the gene-forgene hypothesis. Plant J. 2:359-366.

Van den Ackerveken, G., Marois, E., and Bonas, U. 1996. Recognition of the bacterial avirulence protein AvrBs3 occurs inside the host plant cell. Cell 87:1307-1316.

Van der Hoorn, R. A. L., Laurent, F., Roth, R., and De Wit, P. J. G. M. 2000. Agroinfiltration is a versatile tool that facilitates comparative analyses of $A v r 9 / C f$-9-induced and $A v r 4 / C f$-4-induced necrosis. Mol Plant-Microbe Interact. 13:439-446.

van Kan, J. A. L., Van den Ackerveken, G. F. J. M., and de Wit, P. J. G. M. 1991. Cloning and characterization of cDNA of avirulence gene avr9 of the fungal pathogen Cladosporium fulvum, causal agent of tomato leaf mold. Mol. Plant-Microbe Interact. 4:52-59. 